\title{
CARTESIAN CLOSED EXACT COMPLETIONS IN TOPOLOGY
}

\author{
MARIA MANUEL CLEMENTINO, DIRK HOFMANN, AND WILLIAN RIBEIRO
}

\begin{abstract}
Using generalized enriched categories, in this paper we show that Rosický's proof of cartesian closedness of the exact completion of the category of topological spaces can be extended to a wide range of topological categories over Set, like metric spaces, approach spaces, ultrametric spaces, probabilistic metric spaces, and bitopological spaces. In order to do so we prove a sufficient criterion for exponentiability of $(\mathbb{T}, V)$-categories and show that, under suitable conditions, every injective $(\mathbb{T}, V)$-category is exponentiable in $(\mathbb{T}, V)$-Cat.
\end{abstract}

\section{INTRODUCTION}

As Lawvere has shown in his celebrated paper [Law73], when $V$ is a closed category the category $V$-Cat of $V$-enriched categories and $V$-functors is also monoidal closed. This result extends neither to the cartesian structure nor to the more general setting of $(\mathbb{T}, V)$-categories. Indeed, cartesian closedness of $V$ does not guarantee cartesian closedness of $V$-Cat: take for instance the category of (Lawvere's) metric spaces $P_{+-}$Cat, where $P_{+}$is the complete real half-line, ordered with the $\geq$ relation, and equipped with the monoidal structure given by addition $+; P_{+}$is cartesian closed but $P_{+}$-Cat is not (see [CH06] for details); and, even when the monoidal structure of $V$ is the cartesian one, the category $(\mathbb{T}, V)$-Cat of $(\mathbb{T}, V)$-categories and $(\mathbb{T}, V)$-functors (see [CT03]) does not need to be cartesian closed, as it is the case of the category Top of topological spaces and continuous maps, that is $(\mathbb{U}, 2)$-Cat for $\mathbb{U}$ the ultrafilter monad.

Rosický showed in Ros99] that Top is weakly cartesian closed, and, consequently, that its exact completion is cartesian closed. Weak cartesian closedness of Top follows from the existence of enough injectives in its full subcategory $\mathbf{T o p}_{0}$ of $T 0$-spaces and the fact that they are exponentiable, and this feature, together with several good properties of Top, gives cartesian closedness of its exact completion. More precisely, Rosický has shown in [Ros99] the following theorem.

Theorem 1.1. Let $\mathbf{C}$ be a complete, infinitely extensive and well-powered category with (reg epi, mono)-factorizations such that $f \times 1$ is an epimorphism whenever $f$ is a regular epimorphism. Then the exact completion of $\mathbf{C}$ is cartesian closed provided that $\mathbf{C}$ is weakly cartesian closed.

In this paper we use the setting of $(\mathbb{T}, V)$-categories, for a quantale $V$ and a Set-monad $\mathbb{T}$ laxly extended to $V$-Rel to conclude, in a unified way, that several topological categories over Set share with Top the cartesian closedness of the exact completion. This was recently used by Adámek and Rosický in the study of free completions of categories [AR18]. In fact, the category ( $\mathbb{T}, V)$-Cat is topological over Set [CH03, CT03, hence complete and with (reg epi, mono)-factorizations such that $f \times 1$ is an epimorphism whenever $f$ is, and it is infinitely extensive [MST06. To assure weak

2010 Mathematics Subject Classification. 18B30, 18B35, 18D15, 18D20, 54B30, 54E35, 54E70.

Key words and phrases. quantale, enriched category, (probabilistic) metric space, exponentiation, (weakly) cartesian closed category, exact completion.

Research partially supported by Centro de Matemática da Universidade de Coimbra - UID/MAT/00324/2019, by Centro de Investigação e Desenvolvimento em Matemática e Aplicações da Universidade de Aveiro/FCT UID/MAT/04106/2019, funded by the Portuguese Government through FCT/MCTES and co-funded by the European Regional Development Fund through the Partnership Agreement PT2020. W. Ribeiro also acknowledges the FCT PhD Grant PD/BD/128059/2016. 
cartesian closedness of $(\mathbb{T}, V)$-Cat we consider two distinct scenarios, either restricting to the case that $V$ is a frame - so that its monoidal structure is the cartesian one - or considering the case that the lax extension is determined by a $\mathbb{T}$-algebraic structure on $V$, as introduced in Hof07. under the name of topological theory. In the latter case the proof generalizes Rosický's proof for $\mathbf{T o p}_{0}$, after observing that, using the Yoneda embedding of [CH09, Hof11], every separated $(\mathbb{T}, V)$-category can be embedded in an injective one, and, moreover, these are exponentiable in $(\mathbb{T}, V)$-Cat. For general $(\mathbb{T}, V)$-categories one proceeds again as in [Ros99], using the fact that the reflection of $(\mathbb{T}, V)$-Cat into its full subcategory of separated $(\mathbb{T}, V)$-categories preserves finite products. As observed by Rosický, the exact completion of Top relates to the cartesian closed category of equilogical spaces [BBS04]. Analogously, our approach leads to the study of generalized equilogical spaces, as developed in [Rib18].

The paper is organized as follows. In Section 2 we introduce $(\mathbb{T}, V)$-categories and list their properties used throughout the paper. In Section 3 we revisit the exponentiability problem in $(\mathbb{T}, V)$-Cat, establishing a sufficient criterion for exponentiability which generalizes the results obtained in [Hof07, HS15]. In Section 4 we study the properties of injective $(\mathbb{T}, V)$-categories which will be used in the forthcoming section to conclude that, under suitable assumptions, injective $(\mathbb{T}, V$ )-categories are exponentiable (Theorem 5.8). This result will allow us to conclude, in Theorem 6.3 that $(\mathbb{T}, V)$-Cat is weakly cartesian closed, and, finally, thanks to Theorem 1.1 , that the exact completion of $(\mathbb{T}, V)$-Cat is cartesian closed. We conclude our paper with a section on examples, which include, among others, metric spaces, approach spaces, probabilistic metric spaces, and bitopological spaces.

\section{The CATEGORY of (T, $V$ )-CATEGORIES}

Throughout $V$ is a commutative and unital quantale, i.e $V$ is a complete lattice with a symmetric and associative tensor product $\otimes$, with unit $k$ and right adjoint hom, so that $u \otimes v \leq w$ if, and only if, $v \leq \operatorname{hom}(u, w)$, for all $u, v, w \in V$. Further assume that $V$ is a Heyting algebra, so that $u \wedge-$ also has a right adjoint, for every $u \in V$. We denote by $V$-Rel the 2-category of $V$-relations (or $V$-matrices), having as objects sets, as 1-cells $V$-relations $r: X \rightarrow Y$, i.e. maps $r: X \times Y \rightarrow V$, and 2-cells $\varphi: r \rightarrow r^{\prime}$ given by componentwise order $r(x, y) \leq r^{\prime}(x, y)$. Composition of 1-cells is given by relational composition. $V$-Rel has an involution, given by transposition: the transpose of $r: X \rightarrow Y$ is $r^{\circ}: Y \rightarrow X$ with $r^{\circ}(y, x)=r(x, y)$.

We fix a non-trivial monad $\mathbb{T}=(T, m, e)$ on Set satisfying $(B C)$, i.e. $T$ preserves weak pullbacks and the naturality squares of the natural transformation $m$ are weak pullbacks (see [CHJ14]). In general we do not assume that $T$ preserves products. Later we will make use of the comparison map $\operatorname{can}_{X, Y}: T(X \times Y) \rightarrow T X \times T Y$ defined by $\operatorname{can}_{X, Y}(\mathfrak{w})=\left(T \pi_{X}(\mathfrak{w}), T \pi_{Y}(\mathfrak{w})\right)$ for all $\mathfrak{w} \in T(X \times Y)$, where $\pi_{X}$ and $\pi_{Y}$ are the product projections. Moreover, we assume that $\mathbb{T}$ has an extension to $V$-Rel, which we also denote by $\mathbb{T}$, in the following sense:

- there is a lax functor $T: V$-Rel $\rightarrow V$-Rel which extends $T:$ Set $\rightarrow$ Set;

$-T\left(r^{\circ}\right)=(T r)^{\circ}$ for all $V$-relations $r$; 
- the natural transformations $e: 1_{V \text {-Rel }} \rightarrow T$ and $m: T^{2} \rightarrow T$ become op-lax; that is, for every $r: X \rightarrow Y$,

$$
\begin{gathered}
e_{Y} \cdot r \leq T r \cdot e_{X}, \\
X \stackrel{e_{X}}{\longrightarrow} T X \\
r \quad \leq \quad{ }^{\downarrow} T r \\
\downarrow-\underset{e_{Y}}{\longrightarrow} T Y
\end{gathered}
$$$$
m_{Y} \cdot \operatorname{Tr} \leq \operatorname{Tr} \cdot m_{X}
$$

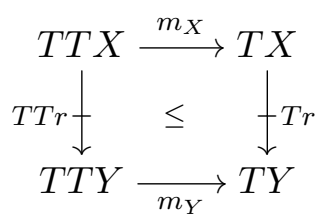

We note that our conditions are stronger than those used in [HST14].

A $(\mathbb{T}, V)$-category is a pair $(X, a)$ where $X$ is a set and $a: T X \rightarrow X$ is a $V$-relation such that

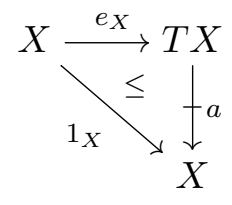

and

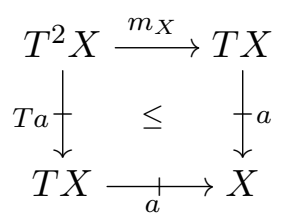

that is, the map $a: T X \times X \rightarrow V$ satisfies the conditions:

(R) for each $x \in X, k \leq a\left(e_{X}(x), x\right)$;

(T) for each $\mathfrak{X} \in T^{2} X, \mathfrak{x} \in T X, x \in X, T a(\mathfrak{X}, \mathfrak{x}) \otimes a(\mathfrak{x}, x) \leq a\left(m_{X}(\mathfrak{X}), x\right)$.

Given $(\mathbb{T}, V)$-categories $(X, a),(Y, b)$, a $(\mathbb{T}, V)$-functor $f:(X, a) \rightarrow(Y, b)$ is a map $f: X \rightarrow Y$ such that

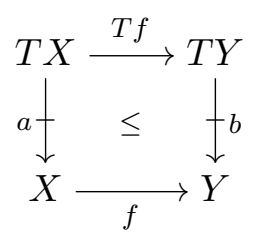

that is, for each $\mathfrak{x} \in T X$ and $x \in X, a(\mathfrak{x}, x) \leq b(T f(\mathfrak{x}), f(x)) ; f$ is said to be fully faithful when this inequality is an equality.

$(\mathbb{T}, V)$-categories and $(\mathbb{T}, V)$-functors form the category $(\mathbb{T}, V)$-Cat. If $(X, a: T X \rightarrow X)$ satisfies $(\mathrm{R})$ (and not necessarily $(\mathrm{T})$ ), we call it a $(\mathbb{T}, V)$-graph. The category $(\mathbb{T}, V)$-Gph, of $(\mathbb{T}, V)$-graphs and $(\mathbb{T}, V)$-functors, contains $(\mathbb{T}, V)$-Cat as a full reflective subcategory.

We present the examples in detail in the last section. We mention here, however, that the leading examples are obtained when one considers the quantale $2=(\{0,1\}, \leq, \&, 1)$ and Lawvere's real half-line $P_{+}=([0, \infty], \geq,+, 0)$, the identity monad $\mathbb{I}$ and the ultrafilter monad $\mathbb{U}$ on Set. Thus we obtain the following examples:

- (II, $V$ )-Cat is the category of $V$-categories and $V$-functors; in particular, (I, 2)-Cat is the category Ord of (pre)ordered sets and monotone maps, while $\left(\mathbb{I}, P_{+}\right)$-Cat is the category Met of Lawvere's metric spaces and non-expansive maps (see [Law73]).

- (UU, 2)-Cat is the category Top of topological spaces and continuous maps.

- $\left(\mathbb{U}, P_{+}\right)$-Cat is the category App of Lowen's approach spaces and non-expansive maps (see [Low97]).

We recall (see AHS90, Definition 21.1]) that a functor $G: \mathbf{A} \rightarrow \mathbf{B}$ is said to be topological if every source $\left(f_{i}: B \rightarrow G A_{i}\right)_{i \in I}$ in $\mathbf{B}$ has a unique $G$-initial lift $\left(\overline{f_{i}}: A \rightarrow A_{i}\right)_{i \in I}$. The following was proved in [CH03] (see also CT03]).

Theorem 2.1. The forgetful functors $(\mathbb{T}, V)$-Cat $\rightarrow$ Set and $(\mathbb{T}, V)$-Gph $\rightarrow$ Set are topological.

This shows, in particular, that (see [AHS90, Chapter 21] for details):

- $(\mathbb{T}, V)$-Cat is complete and cocomplete. 
- Monomorphisms in (T, $V$ )-Cat are the morphisms whose underlying map is injective; therefore, since the $(\mathbb{T}, V)$-structures on any set form a set, $(\mathbb{T}, V)$-Cat is well-powered.

- Every topological category over Set has two factorization systems, (reg epi, mono) and (epi, reg mono); in ( $\mathbb{T}, V)$-Cat the former one is in general not stable (that is, regular epimorphisms need not be stable under pullback - Top is such an example), but the latter one is. Indeed, epimorphisms in $(\mathbb{T}, V)$-Cat are the $(\mathbb{T}, V)$-functors which are surjective as maps, the forgetful functor $(\mathbb{T}, V)$-Cat $\rightarrow$ Set preserves pullbacks, and surjective maps are stable under pullback in Set. Therefore, as $f \times 1_{Z}$ is the pullback of $f: X \rightarrow Y$ along $\pi_{Y}: Y \times Z \rightarrow Y$, we conclude that $f \times 1_{Z}$ is an epimorphism provided $f$ is.

$(\mathbb{T}, V)$-Cat has a natural structure of 2-category: for $(\mathbb{T}, V)$-functors $f, g:(X, a) \rightarrow(Y, b), f \leq g$ if $g \cdot a \leq b \cdot T f$. This condition can be equivalently written as $k \leq b\left(e_{Y}(f(x)), g(x)\right)$ for every $x \in X$ (see [CT03] for details). We write $f \simeq g$ if $f \leq g$ and $g \leq f$.

Extensivity of $(\mathbb{T}, V)$-Cat was studied in [MST06]:

Theorem 2.2. ( $\mathbb{T}, V)$-Cat is infinitely extensive.

In general $(\mathbb{T}, V)$-Cat is not cartesian closed, while $(\mathbb{T}, V)$-Gph is. In fact, the following was proved in [CHT03]:

Theorem 2.3. ( $\mathbb{T}, V)$-Gph is a quasi-topos.

We also note that the tensor product of $V$ induces a canonical structure $c$ on $X \times Y$ defined by

$$
c(\mathfrak{w},(x, y))=a\left(T \pi_{X}(\mathfrak{w}), x\right) \otimes b\left(T \pi_{Y}(\mathfrak{w}), y\right),
$$

where $\mathfrak{w} \in T(X \times Y), x \in X, y \in Y$. We put

$$
(X, a) \otimes(Y, b)=(X \times Y, c),
$$

and this construction is in an obvious way part of a functor $\otimes:(\mathbb{T}, V)$ - $\mathbf{G p h} \times(\mathbb{T}, V)-\mathbf{G p h} \rightarrow$ $(\mathbb{T}, V)$-Gph. However, the tensor product of two $(\mathbb{T}, V)$-categories is in general not a $(\mathbb{T}, V)$ category (see Hof07, Lemma 6.1]).

Weak cartesian closedness of $(\mathbb{T}, V)$-Cat needs a thorough study of injective $(\mathbb{T}, V)$-categories and some extra conditions. This is the subject of the following sections.

\section{Exponentiable $(\mathbb{T}, V)$-CAtegories}

Recall that an object $C$ of a category $\mathbf{C}$ with finite products is exponentiable whenever the functor $C \times-: \mathbf{C} \rightarrow \mathbf{C}$ has a right adjoint. The category $\mathbf{C}$ is cartesian closed if every object $C$ of $\mathbf{C}$ is exponentiable. Equivalently, if for each pair of objects $A, B$ of $\mathbf{C}$ there exists an object $\langle A, B\rangle$ and a morphism ev : $\langle A, B\rangle \times A \rightarrow B$ such that, for each morphism $f: C \times A \rightarrow B$ there exists a unique morphism $\bar{f}: C \rightarrow\langle A, B\rangle$ with ev $\cdot\left(\bar{f} \times 1_{A}\right)=f$. Dropping uniqueness of $\bar{f}$ gives the notion of weakly cartesian closed category.

In this section we present a sufficient condition for a $(\mathbb{T}, V)$-category $X$ to be exponentiable in $(\mathbb{T}, V)$-Cat, which generalises [Hof06, Theorem 4.3] and [Hof07, Theorem 6.5]. To start, we recall that $(\mathbb{T}, V)$-Cat can be fully embedded into the cartesian closed category $(\mathbb{T}, V)$-Gph. Here, for $(\mathbb{T}, V)$-graphs $(X, a)$ and $(Y, b)$, the exponential $\langle(X, a),(Y, b)\rangle$ has as underlying set

$$
Z:=\left\{h:(X, a) \times\left(1, e_{1}^{\circ}\right) \rightarrow(Y, b) \mid h \text { is a }(\mathbb{T}, V) \text {-functor }\right\},
$$

which becomes a $(\mathbb{T}, V)$-graph when equipped with the largest structure $b^{a}$ making the evaluation map

$$
\mathrm{ev}: Z \times X \rightarrow Y,(h, x) \mapsto h(x)
$$


a (T, $V)$-functor: for $\mathfrak{p} \in T Z$ and $h \in Z$, put

$$
b^{a}(\mathfrak{p}, h)=\bigvee\left\{v \in V \mid \forall \mathfrak{q} \in\left(T \pi_{Z}\right)^{-1}(\mathfrak{p}), x \in X . a\left(T \pi_{X}(\mathfrak{q}), x\right) \wedge v \leq b(T \mathrm{ev}(\mathfrak{q}), h(x))\right\}
$$

where $\pi_{X}$ and $\pi_{Z}$ are the product projections. Note that the supremum above is even a maximum since $-\wedge-$ distributes over suprema.

Given $V$-relations $r: X \rightarrow X^{\prime}$ and $s: Y \rightarrow Y^{\prime}$, we define in $V$-Rel $r \otimes s: X \times Y \rightarrow X^{\prime} \times Y^{\prime}$ by $(r \otimes s)\left((x, y),\left(x^{\prime}, y^{\prime}\right)\right)=r\left(x, x^{\prime}\right) \wedge s\left(y, y^{\prime}\right)$. That is, $r \otimes s=\left(\pi_{X^{\prime}}^{\circ} \cdot r \cdot \pi_{X}\right) \wedge\left(\pi_{Y^{\prime}}^{\circ} \cdot s \cdot \pi_{Y}\right)$ in the ordered set $V-\operatorname{Rel}\left(X \times Y, X^{\prime} \times Y^{\prime}\right)$.

Theorem 3.1. Assume that the diagram

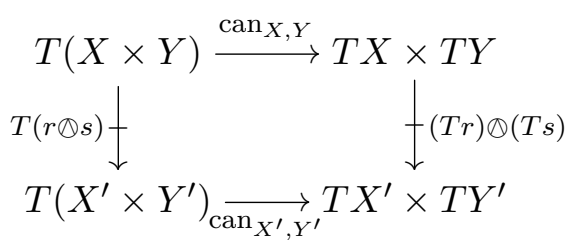

commutes, for all $V$-relations $r: X \rightarrow X^{\prime}$ and $s: Y \rightarrow Y^{\prime}$. Then a $(\mathbb{T}, V)$-category $(X, a)$ is exponentiable provided that

$$
\bigvee_{\mathfrak{x} \in T X}(T a(\mathfrak{X}, \mathfrak{x}) \wedge u) \otimes(a(\mathfrak{x}, x) \wedge v) \geq a\left(m_{X}(\mathfrak{X}), x\right) \wedge(u \otimes v)
$$

for all $\mathfrak{X} \in T T X, x \in X$ and $u, v \in V$.

Proof. We show that the $(\mathbb{T}, V)$-graph structure $b^{a}$ on $Z$ is transitive, for each $(\mathbb{T}, V)$-category $(Y, b)$. To this end, let $\mathfrak{P} \in T T Z, \mathfrak{p} \in T Z, h \in Z, x \in X$ and $\mathfrak{w} \in T(Z \times X)$ with $T \pi_{Z}(\mathfrak{w})=m_{Z}(\mathfrak{P})$. We have to show that

$$
\left(T\left(b^{a}\right)(\mathfrak{P}, \mathfrak{p}) \otimes b^{a}(\mathfrak{p}, h)\right) \wedge a\left(T \pi_{X}(\mathfrak{w}), x\right) \leq b(T \operatorname{ev}(\mathfrak{w}), h(x))
$$

Since $m$ has $(\mathrm{BC})$, there is some $\mathfrak{Q} \in T T(Z \times X)$ with $T T \pi_{Z}(\mathfrak{Q})=\mathfrak{P}$ and $m_{Z \times X}(\mathfrak{Q})=\mathfrak{w}$. Hence, $m_{X}\left(T T \pi_{X}(\mathfrak{Q})\right)=T \pi_{X}(\mathfrak{w})$, and we calculate:

$$
\begin{aligned}
\left(T\left(b^{a}\right)\right. & \left.(\mathfrak{P}, \mathfrak{p}) \otimes b^{a}(\mathfrak{p}, h)\right) \wedge a\left(T \pi_{X}(\mathfrak{w}), x\right) \\
& \leq \bigvee_{\mathfrak{x} \in T X}\left(\left(T\left(b^{a}\right)\left(T T \pi_{Z}(\mathfrak{Q}), \mathfrak{p}\right) \wedge T a\left(T T \pi_{X}(\mathfrak{Q}), \mathfrak{x}\right)\right) \otimes\left(b^{a}(\mathfrak{p}, h) \wedge a(\mathfrak{x}, x)\right) \quad\right. \text { (by (3.ii)) } \\
& \left.\leq \bigvee_{\mathfrak{x} \in T X} \bigvee_{\mathfrak{q} \in \operatorname{can}^{-1}(\mathfrak{p}, \mathfrak{x})} T\left(b^{a} \otimes a\right)\left(T \operatorname{can}_{Z, X}(\mathfrak{Q}), \mathfrak{q}\right) \otimes\left(b^{a} \otimes a\right)\left(\operatorname{can}_{Z, X}(\mathfrak{q}),(h, x)\right) \quad \text { (using (3.i) }\right) \\
& =\bigvee_{\mathfrak{q} \in\left(T \pi_{Z}\right)^{-1}(\mathfrak{p})} T\left(b^{a} \otimes a\right)\left(T \operatorname{can}_{Z, X}(\mathfrak{Q}), \mathfrak{q}\right) \otimes\left(b^{a} \otimes a\right)\left(\operatorname{can}_{Z, X}(\mathfrak{q}),(h, x)\right) \\
& =\bigvee_{\mathfrak{q} \in\left(T \pi_{Z}\right)^{-1}(\mathfrak{p})} T\left(b^{a} \times a\right)(\mathfrak{Q}, \mathfrak{q}) \otimes\left(b^{a} \times a\right)(\mathfrak{q},(h, x)) \\
& \leq \bigvee_{\mathfrak{q} \in\left(T \pi_{Z}\right)^{-1}(\mathfrak{p})} T b(T T \operatorname{ev}(\mathfrak{Q}), T \operatorname{ev}(\mathfrak{q})) \otimes b(T \operatorname{ev}(\mathfrak{q}), h(x)) \\
& \leq b\left(m_{Y} \cdot T T \operatorname{ev}(\mathfrak{Q}), h(x)\right)=b(T \operatorname{ev}(\mathfrak{w}), h(x)) .
\end{aligned}
$$


Remark 3.2. We note that the inequality $\operatorname{can}_{X^{\prime}, Y^{\prime}} \cdot T(r \otimes s) \leq((T r) \otimes(T s)) \cdot \operatorname{can}_{X, Y}$ is automatically true. Firstly, this inequality is equivalent to $T(r \otimes s) \leq \operatorname{can}_{X^{\prime}, Y^{\prime}}^{\circ} \cdot((T r) \oplus(T s)) \cdot \operatorname{can}_{X, Y}$; secondly,

$$
\begin{aligned}
T(r \otimes s) & =T\left(\left(\pi_{X^{\prime}}^{\circ} \cdot r \cdot \pi_{X}\right) \wedge\left(\pi_{Y^{\prime}}^{\circ} \cdot s \cdot \pi_{Y}\right)\right) \\
& \leq T\left(\pi_{X^{\prime}}^{\circ} \cdot r \cdot \pi_{X}\right) \wedge T\left(\pi_{Y^{\prime}}^{\circ} \cdot s \cdot \pi_{Y}\right) \\
& \leq \operatorname{can}_{X^{\prime}, Y^{\prime}}^{\circ} \cdot((T r) \oplus(T s)) \cdot \operatorname{can}_{X, Y} .
\end{aligned}
$$

It is worthwhile to notice that, when $V$ is a frame, that is $\otimes=\wedge$, the condition above is equivalent to

$$
\bigvee_{\mathfrak{x} \in T X} \operatorname{Ta}(\mathfrak{X}, \mathfrak{x}) \wedge a(\mathfrak{x}, x) \geq a\left(m_{X}(\mathfrak{X}), x\right),
$$

for all $\mathfrak{X} \in T T X$ and $x \in X$. Therefore:

Corollary 3.3. When $V$ is a frame and (3.i) commutes for all $V$-relations $r: X \rightarrow X^{\prime}$ and $s: Y \rightarrow Y^{\prime}, a(\mathbb{T}, V)$-category $(X, a)$ is exponentiable provided that

$$
a \cdot m_{X}=a \cdot T a .
$$

\section{InjeCtive AND RePRESEntable ( $\mathbb{T}, V)$-CAtEgories}

In this section we recall an important class of $(\mathbb{T}, V)$-categories, the so-called representable ones. More information on this type of $(\mathbb{T}, V)$-categories can be found in [CCH15, HST14]. We also recall from [CH09, Hof07, Hof11] that every injective ( $\mathbb{T}, V)$-category is representable.

Based on the lax extension of the Set-monad $\mathbb{T}=(T, m, e)$ to $V$-Rel, $\mathbb{T}$ admits a natural extension to a monad on $V$-Cat, in the sequel also denoted by $\mathbb{T}=(T, m, e)$ (see [Tho09]). Here the functor $T: V$-Cat $\rightarrow V$-Cat sends a $V$-category $\left(X, a_{0}\right)$ to $\left(T X, T a_{0}\right)$, and $e_{X}: X \rightarrow T X$ and $m_{X}: T T X \rightarrow T X$ become $V$-functors for each $V$-category $X$. The Eilenberg-Moore algebras for this monad can be described as triples $\left(X, a_{0}, \alpha\right)$ where $\left(X, a_{0}\right)$ is a $V$-category and $(X, \alpha)$ is an algebra for the Set-monad $\mathbb{T}$ such that $\alpha: T\left(X, a_{0}\right) \rightarrow\left(X, a_{0}\right)$ is a $V$-functor. For $\mathbb{T}$ algebras $\left(X, a_{0}, \alpha\right)$ and $\left(Y, b_{0}, \beta\right)$, a map $f: X \rightarrow Y$ is a homomorphism $f:\left(X, a_{0}, \alpha\right) \rightarrow\left(Y, b_{0}, \beta\right)$ precisely if $f$ preserves both structures, that is, whenever $f:\left(X, a_{0}\right) \rightarrow\left(Y, b_{0}\right)$ is a $V$-functor and $f:(X, \alpha) \rightarrow(Y, \beta)$ is a $\mathbb{T}$-homomorphism.

There are canonical adjoint functors

$$
(V \text {-Cat })^{\mathbb{T}} \underset{\frac{T}{M}}{\frac{K}{T^{T}}}(\mathbb{T}, V) \text {-Cat. }
$$

The functor $K$ associates to each $X=\left(X, a_{0}, \alpha\right)$ in $(V \text {-Cat })^{\mathbb{T}}$ the $(\mathbb{T}, V)$-category $K X=(X, a)$, where $a=a_{0} \cdot \alpha$, and keeps morphisms unchanged. Its left adjoint $M:(\mathbb{T}, V)$-Cat $\rightarrow(V \text {-Cat })^{\mathbb{T}}$ sends a $(\mathbb{T}, V)$-category $(X, a)$ to $\left(T X, T a \cdot m_{X}^{\circ}, m_{X}\right)$ and a $(\mathbb{T}, V)$-functor $f$ to $T f$. Via the adjunction $M \dashv K$ one obtains a lifting of the Set-monad $\mathbb{T}=(T, m, e)$ to a monad on $(\mathbb{T}, V)$-Cat, also denoted by $\mathbb{T}=(T, m, e)$.

In this setting we can define 'duals' in $(V \text {-Cat })^{\mathbb{T}}$ and carry them into $(\mathbb{T}, V)$-Cat. Indeed, since $T: V$-Rel $\rightarrow V$-Rel commutes with the involution $(-)^{\circ}:$ for every $\mathbb{T}$-algebra $X=\left(X, a_{0}, \alpha\right)$ also $\left(X, a_{0}^{\circ}, \alpha\right)$ is a $\mathbb{T}$-algebra. Moreover, if $(X, a)$ is a $(\mathbb{T}, V)$-category, we define $X^{\text {op }}$ by mapping $(X, a)$ into $(V \text {-Cat })^{\mathbb{T}}$ via $M$, dualizing the image in $(V \text {-Cat })^{\mathbb{T}}$, and then carrying it back to $(\mathbb{T}, V)$-Cat; that is,

$$
X^{\mathrm{op}}=K\left((M(X, a))^{\mathrm{op}}\right)=\left(T X, m_{X} \cdot(T a)^{\circ} \cdot m_{X}\right) .
$$

Since the monad $\mathbb{T}=(T, m, e)$ on $(\mathbb{T}, V)$-Cat is lax idempotent (i.e, of Kock-Zöberlein type), an algebra structure $\alpha: T X \rightarrow X$ on a $(\mathbb{T}, V)$-category $X$ is left adjoint to the unit $e_{X}: X \rightarrow T X$. 
We call a $(\mathbb{T}, V)$-category $X$ representable whenever $e_{X}: X \rightarrow T X$ has a left adjoint in $(\mathbb{T}, V)$-Cat; equivalently, whenever there is some $(\mathbb{T}, V)$-functor $\alpha: T X \rightarrow X$ with $\alpha \cdot e_{X} \simeq 1_{X}$, since then

$$
e_{X} \cdot \alpha=T \alpha \cdot e_{T X} \geq T \alpha \cdot T e_{X} \simeq 1_{T X} .
$$

However, a left adjoint $\alpha: T X \rightarrow X$ to $e_{X}$ is in general only a pseudo-algebra structure on $X$, that is,

$$
\alpha \cdot e_{X} \simeq 1_{X} \quad \text { and } \quad \alpha \cdot T \alpha \simeq \alpha \cdot m_{X} .
$$

For every representable $(\mathbb{T}, V)$-category $(X, a)$, the structure $a: T X \rightarrow X$ can be decomposed as $a=a_{0} \cdot \alpha$, where $a_{0}=a \cdot e_{X}$ denotes the underlying $V$-category structure.

A $(\mathbb{T}, V)$-category $X$ is injective whenever, for each fully faithful $h: A \rightarrow B$ in $(\mathbb{T}, V)$-Cat and each $(\mathbb{T}, V)$-functor $f: A \rightarrow X$, there is a $(\mathbb{T}, V)$-functor $g: B \rightarrow X$ with $g \cdot h \simeq f$.

Proposition 4.1. Every injective $(\mathbb{T}, V)$-category is representable.

Proof. Let $X$ be an injective $(\mathbb{T}, V)$-category. The $(\mathbb{T}, V)$-functor $e_{X}:(X, a) \rightarrow\left(T X, T a \cdot m_{X}^{\circ} \cdot m_{X}\right)$ is an embedding. Indeed, $e_{X}$ is injective because the monad $T$ is non-trivial, and it is fully faithful:

$$
e_{X}^{\circ} \cdot T a \cdot m_{X}^{\circ} \cdot m_{X} \cdot T e_{X} \leq a \cdot T a \cdot m_{X}^{\circ} \leq a \cdot m_{X} \cdot m_{X}^{\circ} \leq a .
$$

Hence, there is a $(\mathbb{T}, V)$-functor $\alpha: T X \rightarrow X$ with $\alpha \cdot e_{X} \simeq 1_{X}$, and so $X$ is representable.

\section{INJeCtive $(\mathbb{T}, V)$-CATEGORIES ARE EXPONENTIABLE}

In Section 6 we will show that, under some conditions, $(\mathbb{T}, V)$-Cat is weakly cartesian closed. Notably, we will use that every $(\mathbb{T}, V)$-category can be embedded into an injective one; which, by the main result of this section, implies that every $(\mathbb{T}, V)$-category can be embedded into an exponentiable one. We hasten to remark that this is easily seen to be fulfilled for $\mathbb{T}$ being the identity monad, witnessed by the Yoneda embedding (see [Law73])

$$
y_{X}: X \rightarrow P X:=V^{X^{\mathrm{op}}} \text {. }
$$

Here $P X$ is the free cocompletion of $X$; being cocomplete, $P X$ is injective.

To treat the general case, we will consider from now on only extensions of the $\operatorname{monad} \mathbb{T}$ to $V$-Rel given by a $\mathbb{T}$-algebra structure $\xi: T V \rightarrow V$ on $V$, so that we are dealing with a strict topological theory in the sense of [Hof07]. In this case, the extension of $T$ : Set $\rightarrow$ Set to $V$-Rel is defined by

$$
\begin{aligned}
\operatorname{Tr}: T X \times T Y & \rightarrow V \\
(\mathfrak{x}, \mathfrak{y}) & \mapsto \bigvee\left\{\xi \cdot \operatorname{Tr}(\mathfrak{w}) \mid \mathfrak{w} \in T(X \times Y), T \pi_{X}(\mathfrak{w})=\mathfrak{x}, T \pi_{Y}(\mathfrak{w})=\mathfrak{y}\right\}
\end{aligned}
$$

for each $V$-relation $r: X \times Y \rightarrow V$.

In order to obtain a Yoneda embedding, we consider the $\mathbb{T}$-algebra $(V$, hom, $\xi)$ which is mapped by $K$ into the important $(\mathbb{T}, V)$-category $\left(V, \operatorname{hom}_{\xi}\right)$, where $\operatorname{hom}_{\xi}=$ hom $\cdot \xi$ (see Section 4 ). The proof of the following result can be found in [CH09] and [Hof11].

Theorem 5.1. If the extension of $\mathbb{T}$ to $V$-Rel is induced by a strict topological theory, then, for every $(\mathbb{T}, V)$-category $(X, a)$, the $V$-relation $a: T X \rightarrow X$ defines a $(\mathbb{T}, V)$-functor

$$
a: X^{\mathrm{op}} \otimes X \rightarrow\left(V, \operatorname{hom}_{\xi}\right) .
$$

Moreover, the $\otimes$-exponential mate $y_{X}=\ulcorner a\urcorner: X \rightarrow V^{X^{\mathrm{op}}}$ of a is fully faithful, and the (T, $\left.V\right)$ category $P X=V^{X^{\mathrm{op}}}$ is injective.

In fact, this construction defines a functor $P:(\mathbb{T}, V)$-Cat $\rightarrow(\mathbb{T}, V)$-Cat and $y=\left(y_{X}\right)_{X}$ is a natural transformation $y: 1_{(\mathbb{T}, V) \text {-Cat }} \rightarrow P$. 
Since $y_{X}$ is fully faithful, when $X$ is injective there exists a $(\mathbb{T}, V)$-functor $\operatorname{Sup}_{X}: P X \rightarrow X$ such that $\operatorname{Sup}_{X} \cdot y_{X} \simeq 1_{X}$. As shown in [Hof11, Theorem 2.7], $\operatorname{Sup}_{X} \dashv y_{X}$. Moreover, for each (T, $\left.V\right)$ category $(X, a), y_{X}$ is one-to-one if, and only if, $(X, a)$ is separated, i.e. for every $f, g:(Y, b) \rightarrow$ $(X, a), f \simeq g$ only if $f=g$ (see [HT10, for example). It follows immediately that, for an injective $(\mathbb{T}, V)$-functor $f: X \rightarrow Y$ where $Y$ is separated, also $X$ is.

Lemma 5.2. The $\otimes$-exponential $Y^{X}$ is separated, for every separated $(\mathbb{T}, V)$-category $Y$ and every representable $(\mathbb{T}, V)$-category $X$; in particular, $P X$ is separated, for every $(\mathbb{T}, V)$-category $X$.

Proof. See [HT10, Corollary $4.12(2)]$.

Corollary 5.3. Every separated $(\mathbb{T}, V)$-category embeds into an injective $(\mathbb{T}, V)$-category.

In Section 2 we introduced the tensor product $X \otimes Y$ of $(\mathbb{T}, V)$-graphs $X$ and $Y$. We remark that, in the setting of a strict topological theory, $X \otimes Y$ is a $(\mathbb{T}, V)$-category provided that $X$ and $Y$ are so (see [Hof07]).

The result promised in the title of this section was shown in [Hof14, Proposition 2.7] for the special case of $\otimes=\wedge$ :

Proposition 5.4. If the quantale $V$ is a frame and (3.i) commutes for all $V$-relations $r: X \rightarrow X^{\prime}$ and $s: Y \rightarrow Y^{\prime}$, then every representable $(\mathbb{T}, V)$-category is exponentiable. In particular, in this case every injective $(\mathbb{T}, V)$-category is exponentiable.

To treat the general case, we will make use of the following conditions:

Assumptions 5.5. (1) The diagram (3.i) commutes, for all $V$-relations $r: X \rightarrow X^{\prime}$ and $s: Y \rightarrow Y^{\prime}$.

(2) For all $u, v, w \in V$,

$$
w \wedge(u \otimes v)=\bigvee\left\{u^{\prime} \otimes v^{\prime} \mid u^{\prime} \leq u, v^{\prime} \leq v, u^{\prime} \otimes v^{\prime} \leq w\right\} ;
$$

or, equivalently, every injective $V$-category is exponentiable: see [HR13, Theorem 5.3].

(3) For every $V$-relation $a: X \rightarrow Y$ and $u \in V$,

$$
T(a \otimes u)=T a \otimes u,
$$

where $a \otimes u$ is the $V$-relation defined by $(a \otimes u)(x, y)=a(x, y) \otimes u$.

(4) The maps $V \otimes V \stackrel{\otimes}{\longrightarrow} V$ and $X \stackrel{(-, u)}{\longrightarrow} X \otimes V$ are $(\mathbb{T}, V)$-functors, for all $u \in V$.

These morphisms induce an interesting action of $V$ on every injective $(\mathbb{T}, V)$-category $(X, a)$ as follows. The $(\mathbb{T}, V)$-functor

$$
X^{\mathrm{op}} \otimes X \otimes V \stackrel{a \otimes 1}{\longrightarrow} V \otimes V \stackrel{\otimes}{\longrightarrow} V
$$

induces a $(\mathbb{T}, V)$-functor $\tilde{a}: X \otimes V \rightarrow P X$. We denote the composite

$$
X \otimes V \stackrel{\tilde{a}}{\longrightarrow} P X \stackrel{\operatorname{Sup}_{X}}{\longrightarrow} X
$$

by $\oplus$, and

$$
X \stackrel{(-, u)}{\longrightarrow} X \otimes V \stackrel{\tilde{a}}{\longrightarrow} P X \stackrel{\operatorname{Sup}_{X}}{\longrightarrow} X,
$$

assigning to each $x \in X$ an element $x \oplus u$ in $X$, by $-\oplus u$.

Analogously we will write $\mathfrak{x} \oplus u$ for $T(-\oplus u)(\mathfrak{x})$, for every $\mathfrak{x} \in T X$ and $u \in V$. Note that $(\mathbb{T}, V)$-functoriality of $-\oplus u$ can be written as

$$
a(\mathfrak{x}, y) \leq a(\mathfrak{x} \oplus u, y \oplus u),
$$

for every $\mathfrak{x} \in T X$ and $y \in X$. 
Lemma 5.6. Assuming 5.5 (4), for an injective $(\mathbb{T}, V)$-category $(X, a)$, with $a=a_{0} \cdot \alpha$ as usual, the following holds, for every $x, y \in X, \mathfrak{x} \in T X$ and $u \in V$ :

(1) $a_{0}(x \oplus u, y)=\operatorname{hom}\left(u, a_{0}(x, y)\right)$;

(2) $a_{0}(x, y \oplus u) \geq a_{0}(x, y) \otimes u$;

(3) $a(\mathfrak{x} \oplus u, y) \geq \operatorname{hom}(u, a(\mathfrak{x}, y))$;

(4) $a(\mathfrak{x}, y \oplus u) \geq a(\mathfrak{x}, y) \otimes u$.

Moreover, if, in addition, 5.5 (3) holds, then, for every $\mathfrak{X} \in T^{2} X, \mathfrak{y} \in T X, u \in V$,

(5) $T a(\mathfrak{X}, \mathfrak{y} \oplus u) \geq T a(\mathfrak{X}, \mathfrak{y}) \otimes u$.

Proof. (1) For every $x, y \in X$ and $u \in V$,

$$
\begin{aligned}
a_{0}(x \oplus u, y) & =a_{0}\left(\operatorname{Sup}_{X}(\tilde{a}(x, u)), y\right) & & \text { (by definition of } \oplus \text { ) } \\
& =\left[\tilde{a}(x, u), y_{X}(y)\right] & & \left(\text { because } \operatorname{Sup}_{X} \dashv y_{X}\right) \\
& =\bigwedge_{\mathfrak{x} \in T X} \operatorname{hom}\left(\tilde{a}(x, u)(\mathfrak{x}), y_{X}(y)(\mathfrak{x})\right) & & \text { (by definition of }[,]) \\
& =\bigwedge_{\mathfrak{x} \in T X} \operatorname{hom}(a(\mathfrak{x}, x) \otimes u, a(\mathfrak{x}, y)) & & \text { (by definition of } \left.\tilde{a} \text { and } y_{X}(y)\right) \\
& =\operatorname{hom}\left(u, a_{0}(x, y)\right), & &
\end{aligned}
$$

because, using the fact that $a=a_{0} \cdot \alpha$ and

$$
a_{0}(\alpha(\mathfrak{x}), x) \otimes u \otimes \operatorname{hom}\left(u, a_{0}(x, y)\right) \leq a_{0}(\alpha(\mathfrak{x}), x) \otimes a_{0}(x, y) \leq a_{0}(\alpha(\mathfrak{x}), y),
$$

for $\mathfrak{x} \in T X$, we can conclude that

$$
\operatorname{hom}\left(u, a_{0}(x, y)\right) \leq \bigwedge_{\mathfrak{x} \in T X} \operatorname{hom}\left(a_{0}(\alpha(\mathfrak{x}), x) \otimes u, a_{0}(\alpha(\mathfrak{x}), y)\right) .
$$

Taking $\mathfrak{x}=e_{X}(x)$, we see that this inequality is in fact an equality as claimed.

(2) Since, by hypothesis, $-\oplus u$ is a $(\mathbb{T}, V)$-functor, and so, in particular, a $V$-functor $\left(X, a_{0}\right) \rightarrow$ $\left(X, a_{0}\right)$,

$$
a_{0}(x, y) \leq a_{0}(x \oplus u, y \oplus u)=\operatorname{hom}\left(u, a_{0}(x, y \oplus u)\right)
$$

and then

$$
a_{0}(x, y) \otimes u \leq \operatorname{hom}\left(u, a_{0}(x, y \oplus u)\right) \otimes u \leq a_{0}(x, y \oplus u) .
$$

(3) One has

$$
\begin{aligned}
k & \leq a_{0}(\alpha(\mathfrak{x}), \alpha(\mathfrak{x}))=a(\mathfrak{x}, \alpha(\mathfrak{x})) \\
& \leq a(\mathfrak{x} \oplus u, \alpha(\mathfrak{x}) \oplus u) \\
& =a_{0}(\alpha(\mathfrak{x} \oplus u), \alpha(\mathfrak{x}) \oplus u) .
\end{aligned}
$$

Using (1) we conclude that

$$
\begin{aligned}
\operatorname{hom}(u, a(\mathfrak{x}, y)) & =a_{0}(\alpha(\mathfrak{x}) \oplus u, y) \\
& \leq a_{0}(\alpha(\mathfrak{x} \oplus u), \alpha(\mathfrak{x}) \oplus u) \otimes a_{0}(\alpha(\mathfrak{x}) \oplus u, y) \\
& \leq a_{0}(\alpha(\mathfrak{x} \oplus u), y)=a(\mathfrak{x} \oplus u, y) .
\end{aligned}
$$

(4) follows directly from (2), while (5) follows from (4).

Lemma 5.7. Let $\varphi: V \rightarrow W$ be a surjective quantale homomorphism; that is, $\varphi$ preserves the tensor, the neutral element, and suprema. Then, if $V$ satisfies condition 5.5 (2), so does $W$.

Theorem 5.8. Under Assumptions 5.5, every injective ( $\mathbb{T}, V)$-category is exponentiable in $(\mathbb{T}, V)$-Cat. 
Proof. Let $\mathfrak{X} \in T^{2} X, x \in X$ and $u, v \in V$. In order to conclude that

$$
\bigvee_{\mathfrak{x} \in T X}(T a(\mathfrak{X}, \mathfrak{x}) \wedge u) \otimes(a(\mathfrak{x}, x) \wedge v) \geq a\left(m_{X}(\mathfrak{X}), x\right) \wedge(u \otimes v),
$$

we make use of Hypothesis $5.5(2)$. Let $u^{\prime}, v^{\prime} \in V$ with $u^{\prime} \leq u, v^{\prime} \leq v$ and $u^{\prime} \otimes v^{\prime} \leq a\left(m_{X}(\mathfrak{X}), x\right)$. First we note that

$$
\begin{aligned}
\operatorname{Ta}\left(\mathfrak{X}, T \alpha(\mathfrak{X}) \oplus u^{\prime}\right) \wedge u & \geq\left(\operatorname{Ta}(\mathfrak{X}, T \alpha(\mathfrak{X})) \otimes u^{\prime}\right) \wedge u \\
& =\left(T a_{0}(T \alpha(\mathfrak{X}), T \alpha(\mathfrak{X})) \otimes u^{\prime}\right) \wedge u \\
& \geq\left(k \otimes u^{\prime}\right) \wedge u=u^{\prime}
\end{aligned}
$$

and

$$
\begin{aligned}
a\left(T \alpha(\mathfrak{X}) \oplus u^{\prime}, x\right) & \geq \operatorname{hom}\left(u^{\prime}, a(T \alpha(\mathfrak{X}), x)\right) \\
& =\operatorname{hom}\left(u^{\prime}, a_{0}(\alpha(T \alpha(\mathfrak{X})), x)\right) \\
& =\operatorname{hom}\left(u^{\prime}, a_{0}\left(\alpha\left(m_{X}(\mathfrak{X})\right), x\right)\right) \\
& =\operatorname{hom}\left(u^{\prime}, a\left(m_{X}(\mathfrak{X}), x\right)\right) .
\end{aligned}
$$

Now, from $u^{\prime} \otimes v^{\prime} \leq a\left(m_{X}(\mathfrak{X}), x\right)$ and $v^{\prime} \leq v$ we get

$$
v^{\prime} \leq \operatorname{hom}\left(u^{\prime}, a\left(m_{X}(\mathfrak{X}), x\right)\right) \wedge v \leq a\left(T \alpha(\mathfrak{X}) \oplus u^{\prime}, x\right) \wedge v,
$$

hence

$$
u^{\prime} \otimes v^{\prime} \leq\left(T a\left(\mathfrak{X}, T \alpha(\mathfrak{X}) \oplus u^{\prime}\right) \wedge u\right) \otimes\left(a\left(T \alpha(\mathfrak{X}) \oplus u^{\prime}, x\right) \wedge v\right) .
$$

Therefore $a\left(m_{X}(\mathfrak{X}), x\right) \wedge(u \otimes v) \leq \bigvee_{\mathfrak{x} \in T X}(T a(\mathfrak{X}, \mathfrak{x}) \wedge u) \otimes(a(\mathfrak{x}, x) \wedge v)$.

Remark 5.9. Under Assumptions 5.5, it follows from Lemma 5.2 that the exponential $\langle(X, a),(Y, b)\rangle$ is separated, for all separated injective $(\mathbb{T}, V)$-categories $(X, a)$ and $(Y, b)$. In fact, with $a=a_{0} \cdot \alpha$, the epimorphism $(X, \alpha) \rightarrow(X, a)$ in $(\mathbb{T}, V)$-Cat is mapped to the monomorphism

$$
\langle(X, a),(Y, b)\rangle \quad \longrightarrow \quad\langle(X, \alpha),(Y, b)\rangle=(Y, b)^{(X, \alpha)},
$$

which proves that $\langle(X, a),(Y, b)\rangle$ is separated.

\section{6. (T, $V$ )-Cat IS WEAKLy CARTESIAN CLOSED}

Building on the results of the previous section, in this section we show that, under some conditions, $(\mathbb{T}, V)$-Cat is weakly cartesian closed. We start by proving this property for the full subcategory $(\mathbb{T}, V)$-Cat $\mathbf{t}_{\text {sep }}$ of $(\mathbb{T}, V)$-Cat of separated $(\mathbb{T}, V)$-categories.

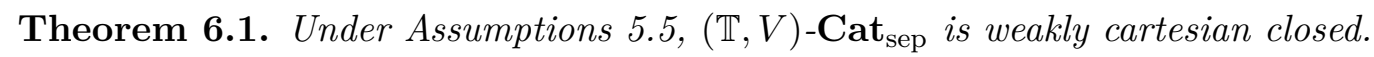

Proof. For $X, Y$ separated (T, $V$ )-categories, consider the Yoneda embeddings $y_{X}: X \rightarrow P X$ and $y_{Y}: Y \rightarrow P Y$, and the exponential $\langle P X, P Y\rangle$. The elements of its underlying set can be identified with $\left(\mathbb{T}, V\right.$ )-functors $E \times P X \rightarrow P Y$ (where $E=\left(1, e_{1}^{\circ}\right)$ is the generator of $(\mathbb{T}, V)$-Cat), and the universal morphism ev : $\langle P X, P Y\rangle \times P X \rightarrow P Y$ with the evaluation map: $\operatorname{ev}(\varphi, \mathfrak{x})=\varphi(\mathfrak{x})$ (where, for simplicity, we identify the set $E \times P X$ with $P X)$. We can therefore consider

$$
\ll X, Y \gg=\left\{\varphi: E \times P X \rightarrow P Y \mid \varphi\left(y_{X}(X)\right) \subseteq y_{Y}(Y)\right\}
$$

with the initial structure with respect to the inclusion $\iota: \ll X, Y \gg \rightarrow\langle P X, P Y\rangle$. Moreover, the morphism

$$
\ll X, Y \gg \times X \stackrel{\iota \times y_{X}}{\longrightarrow}\langle P X, P Y\rangle \times P X \stackrel{\text { ev }}{\longrightarrow} P Y
$$


factors through $y_{Y}$ via a morphism

$$
\ll X, Y \gg \times X \underset{\mathrm{ev}}{\longrightarrow} Y .
$$

Next we show that this is a weak exponential in $(\mathbb{T}, V)-\mathbf{C a t}_{\text {sep }}$.

Given any separated $(\mathbb{T}, V)$-category $Z$, and a $(\mathbb{T}, V)$-functor $f: Z \times X \rightarrow Y$, by injectivity of $P Y$ there exists a $(\mathbb{T}, V)$-functor $f^{\prime}: Z \times P X \rightarrow P Y$ making the square below commute. Then, by universality of the evaluation map ev, there exists a unique $(\mathbb{T}, V)$-functor $\bar{f}: Z \rightarrow\langle P X, P Y\rangle$ making the bottom triangle commute.

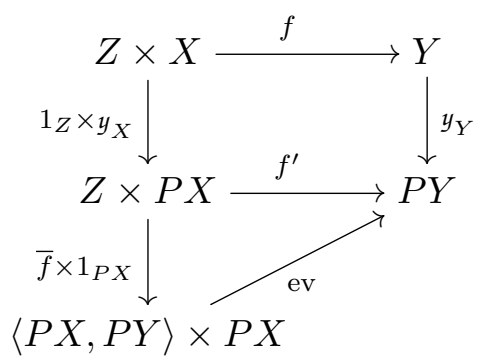

The map $\bar{f}: Z \rightarrow\langle P X, P Y\rangle$, assigning to each $z \in Z$ a map $\bar{f}(z): P X \rightarrow P Y$, is such that $\bar{f}(z)\left(y_{X}(x)\right)=\operatorname{ev}\left(\bar{f}(z), y_{X}(x)\right)=y_{Y}(f(z, x))$; that is, $\bar{f}(z)\left(y_{X}(X)\right) \subseteq y_{Y}(Y)$, and this means that $\bar{f}(z) \in \ll X, Y \gg$. Hence we can consider the corestriction $\tilde{f}$ of $\bar{f}$ to $\ll X, Y \gg$, which is again a $(\mathbb{T}, V)$-functor since $\ll X, Y \gg$ has the initial structure with respect to $\langle P X, P Y\rangle$, so that the following diagram commutes.

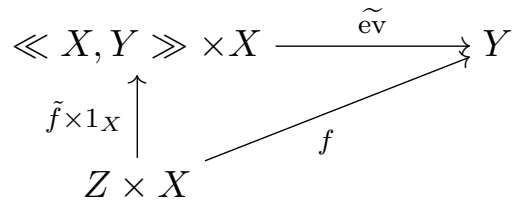

In order to show that $(\mathbb{T}, V)$-Cat is weakly cartesian closed, we follow the proof of Ros99. Hence, first we show that:

Proposition 6.2. The reflector $R:(\mathbb{T}, V)$-Cat $\rightarrow(\mathbb{T}, V)-\mathbf{C a t}_{\mathrm{sep}}$ preserves finite products.

Proof. We recall that, for any $(\mathbb{T}, V)$-category $(X, a), R(X, a)=(\tilde{X}, \tilde{a})$, with $\tilde{X}=X / \sim$, where $x \sim y$ if $k \leq a\left(e_{X}(x), y\right) \wedge a\left(e_{X}(y), x\right)$, and $\tilde{a}=\eta_{X} \cdot a \cdot\left(T \eta_{X}\right)^{\circ}$, with $\eta_{X}: X \rightarrow \tilde{X}$ the projection. This structure makes $\eta_{X}$ both an initial and a final morphism (see [HST14] for details).

Let $f: R(X \times Y) \rightarrow R X \times R Y$ be the unique morphism such that $f \cdot \eta_{X \times Y}=\eta_{X} \times \eta_{Y}$.

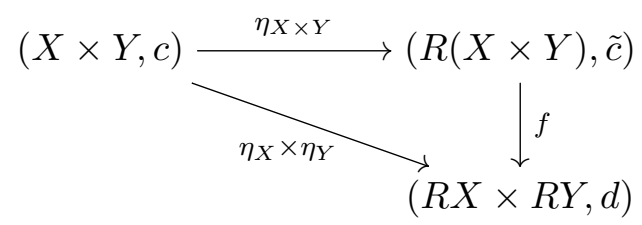

From $c\left(e_{X \times Y}(x, y),\left(x^{\prime}, y^{\prime}\right)\right)=a\left(e_{X}(x), x^{\prime}\right) \wedge b\left(e_{Y}(y), y^{\prime}\right)$ it is immediate that $(x, y) \sim\left(x^{\prime}, y^{\prime}\right)$ in $X \times Y$ if, and only if, $x \sim x^{\prime}$ in $X$ and $y \sim y^{\prime}$ in $Y$. Therefore, $f$ is a bijection. Assuming the Axiom of Choice, so that $T$ preserves surjections, we have, for every $\mathfrak{z} \in T(R(X \times Y)),(x, y) \in X \times Y$,

$$
\begin{aligned}
\tilde{c}(\mathfrak{z},[(x, y)]) & =c(\mathfrak{w},(x, y)) & & \left(\text { for any } \mathfrak{w} \in\left(T \eta_{X \times Y}\right)^{-1}(\mathfrak{z})\right) \\
& =d\left(T\left(\eta_{X} \times \eta_{Y}\right)(\mathfrak{w}),([x],[y])\right) & & \text { (because } \eta_{X} \times \eta_{Y} \text { is initial) } \\
& =d(T f(\mathfrak{z}),([x],[y]) ; & &
\end{aligned}
$$

that is, $f$ is initial and therefore an isomorphism. 
Theorem 6.3. Under Assumptions 5.5, (T, V)-Cat is weakly cartesian closed.

Proof. Given $(\mathbb{T}, V)$-categories $(X, a),(Y, b)$, to build the weak exponential $\ll X, Y \gg$ we will show the cosolution set condition for the functor $-\times(X, a)$.

For each $(\mathbb{T}, V)$-functor $f:(Z, c) \times(X, a) \rightarrow(Y, b)$ we consider its reflection $R f: R Z \times R X \cong$ $R(Z \times X) \rightarrow R Y$ and we factorise it through the weak evaluation in $(\mathbb{T}, V)-$ Cat $_{\mathrm{sep}}, R f=\widetilde{\mathrm{ev}} \cdot(\overline{R f} \times$ $\left.1_{R X}\right)$, so that in the diagram below the outer rectangle commutes.

Then we define $Z_{f}=Z / \sim$ by

$$
z \sim z^{\prime} \text { if both } f(z, x)=f\left(z^{\prime}, x\right) \text {, for every } x \in X \text {, and } \overline{R f}\left(\eta_{Z}(z)\right)=\overline{R f}\left(\eta_{Z}\left(z^{\prime}\right)\right),
$$

and equip it with the final structure for the projection $q_{f}: Z \rightarrow Z_{f}$. Then $h_{f}: Z_{f} \rightarrow \ll R X, R Y \gg$, with $h_{f}([z])=\overline{R f}\left(\eta_{Z}(z)\right)$, is a $(\mathbb{T}, V)$-functor since its composition with $q_{f}$ is $\overline{R f} \cdot \eta_{Z}$ and $q_{f}$ is final. Then we factorise $f$ via the surjection $q_{f} \times 1_{X}: Z \times X \rightarrow Z_{f} \times X$ as in the diagram below. Moreover, the map $\hat{f}: Z_{f} \times X \rightarrow Y$, with $\hat{f}([z], x)=f(z, x)$, is a $(\mathbb{T}, V)$-functor because $\eta_{Y} \cdot \hat{f}=\widetilde{\mathrm{ev}} \cdot\left(h_{f} \times \eta_{X}\right)$ is and $\eta_{Y}$ is initial.

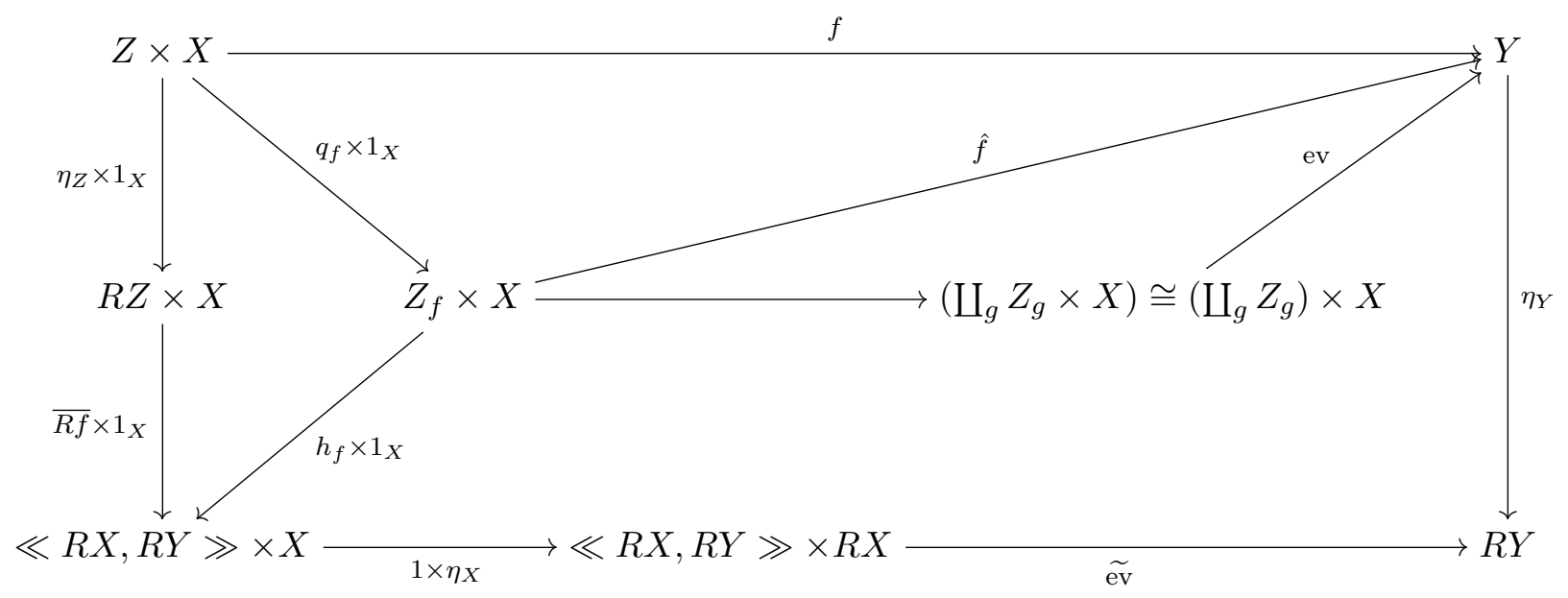

Since the cardinality of $Z_{f}$ is bounded by the cardinality of the set $|\ll R X, R Y \gg| \times|Y|^{|X|}$, as witnessed by the injective map

$$
\begin{aligned}
& Z_{f} \rightarrow|\ll R X, R Y \gg| \times|Y|^{|X|}, \\
& {[z] \mapsto\left(\overline{R f}\left(\eta_{Z}(z)\right), f(z,-)\right)}
\end{aligned}
$$

there is only a set of possible $(\mathbb{T}, V)$-categories $Z_{f}$. Hence we can form its coproduct, as in the diagram above, and consider the induced ( $\mathbb{T}, V$ )-functor ev : $\left(\coprod_{g} Z_{g}\right) \times X \cong \coprod_{g}\left(Z_{g} \times X\right) \rightarrow Y$ (note that the isomorphism follows from extensivity of $(\mathbb{T}, V)$-Cat).

\section{EXAMPLES}

In this section we use Theorem 6.3 to present examples of weakly cartesian closed categories. Hence, in conjunction with the following theorem established in Ros99, we obtain examples of categories with cartesian closed exact completion since all other conditions of that theorem are trivially satisfied in these examples.

Theorem 7.1. Let $\mathbf{C}$ be a complete, infinitely extensive and well-powered category in which every morphism factorizes as a regular epi followed by a mono, and where $f \times 1$ is an epimorphism for every regular epimorphism $f: A \rightarrow B$ in $\mathbf{C}$. Then, if $\mathbf{C}$ is weakly cartesian closed, the exact completion $\mathbf{C}_{\mathrm{ex}}$ of $\mathbf{C}$ is cartesian closed. 
We note that, in order to conclude that $(\mathbb{T}, V)$-Cat is weakly cartesian closed, we have to check whether $V$ and $\mathbb{T}$ satisfy Assumptions 5.5 .

First we analyse examples where $\mathbb{T}$ is the identity monad. In this particular setting we only have to check that 5.5 (2) holds. The category $V$-Cat is always monoidal closed, as shown in Law73. Therefore, when $V$ is a frame considered as a quantale, then $V$-Cat is cartesian closed. This is the case of 2 , and so one concludes that Ord is cartesian closed. Moreover, for $V$ the lattice $([0, \infty], \geq)$ with $\otimes=\wedge, V$-Cat is the category of ultrametric spaces, which is therefore also cartesian closed.

When $V=P_{+}, V$-Cat is the category Met of Lawvere's metric spaces [Law73], which is not cartesian closed (see [CH06] for details). However, the quantale $P_{+}$satisfies 5.5 (2), and so from Theorem 6.3 it follows that Met is weakly cartesian closed.

Metric and ultrametric spaces can be also viewed as categories enriched in a quantale based on the complete lattice $[0,1]$ with the usual "less or equal" relation $\leq$, which is isomorphic to $[0, \infty]$ via the map $[0,1] \rightarrow[0, \infty], u \mapsto-\ln (u)$ where $-\ln (0)=\infty$. More in detail, we consider the following quantale operations on $[0,1]$ with neutral element 1.

(1) For $\otimes=*$ being the ordinary multiplication, via the isomorphism $[0,1] \simeq[0, \infty]$, this quantale is isomorphic to the quantale $P_{+}$, hence $[0,1]$-Cat $\simeq$ Met.

(2) For the tensor $\otimes=\wedge$ being infimum, the isomorphism $[0,1] \simeq[0, \infty]$ establishes an equivalence between $[0,1]$-Cat and the category of ultrametric spaces and non-expansive maps.

(3) Another interesting multiplication on $[0,1]$ is the Eukasiewicz tensor $\otimes=\odot$ given by $u \odot v=\max (0, u+v-1)$. Via the lattice isomorphism $[0,1] \rightarrow[0,1], u \mapsto 1-u$, this quantale is isomorphic to the quantale $[0,1]$ with "greater or equal" relation $\geq$ and tensor $u \otimes v=\min (1, u+v)$ truncated addition. Therefore $[0,1]$-Cat is equivalent to the category of bounded-by-1 metric spaces and non-expansive maps. Moreover, with respect to the "greater or equal" relation and truncated addition on $[0,1]$, the map

$$
[0, \infty] \rightarrow[0,1], u \mapsto \min (1, u)
$$

is a surjective quantale morphism; therefore, by Lemma 5.7, also $[0,1]$ with the Eukasiewicz tensor satisfies 5.5 (2).

(4) More generally, every continuous quantale structure $\otimes$ on the lattice $[0,1]$ (with Euclidean topology and the usual "less or equal" relation) with neutral element 1 satisfies 5.5 (2). This can be shown using the fact, proven in [Fau55] and [MS57, that every such tensor $\otimes:[0,1] \times[0,1] \rightarrow[0,1]$ is a combination of the three operations on $[0,1]$ described above. More precise:

(a) For $u, v \in[0,1]$ and $e \in[0,1]$ idempotent with $u \leq e \leq v: u \otimes v=\min (u, v)=u$.

(b) For every non-idempotent $u \in[0,1]$, there exist idempotents $e$ and $f$ with $e<u<f$ and such that the interval $[e, f]$ (with the restriction of the tensor on $[0,1]$ and with neutral element $f$ ) is isomorphic to $[0,1]$ either with multiplication or Eukasiewicz tensor.

Now let $w, u, v \in[0,1]$. We may assume $u \leq v$. If $u \otimes v \leq w$, then clearly

$$
w \wedge(u \otimes v)=u \otimes v=\bigvee\left\{u^{\prime} \otimes v^{\prime} \mid u^{\prime} \leq u, v^{\prime} \leq v, u^{\prime} \otimes v^{\prime} \leq w\right\} .
$$

We consider now $w<u \otimes v \leq u \leq v$. If $w$ is idempotent, then

$$
w=w \otimes v, \quad w \leq u, \quad v \leq v ;
$$

otherwise there are idempotents $e$ and $f$ with $e<w<f$ and $[e, f]$ is isomorphic to $[0,1]$ either with multiplication or Łukasiewicz tensor.

Case 1: $v \leq f$. Then 5.5 (2) holds since $w, u \otimes v, u, v \in[e, f]$. 
Case 2: $f<v$. Then $w=w \wedge v=w \otimes v, w \leq u$ and $v \leq v$.

We conclude that $[0,1]$-Cat is weakly cartesian closed, for every continuous quantale structure $\otimes$ on the lattice $[0,1]$ with neutral element 1 .

Now let $V=\Delta$ be the quantale of distribution functions (see [HR13, CH17] for details). As observed in [HR13], it verifies 5.5 (2), and so we can conclude from Theorem 6.3 that the category $\Delta$-Cat of probabilistic metric spaces and non-expansive maps is weakly cartesian closed.

When $\mathbb{T}$ is not the identity monad, some further work is need to guarantee Assumptions 5.5 .

Theorem 7.2. (1) The tensor product on the quantale $V$ defines a $(\mathbb{T}, V)$-functor $\otimes: V \otimes V \rightarrow$ $V$.

(2) Let $u \in V$ satisfying $u \cdot ! \geq \xi \cdot T u$.

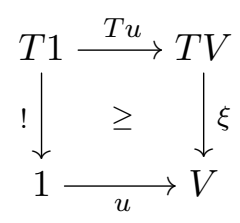

Then $(-, u): X \rightarrow X \times V$ is a $(\mathbb{T}, V)$-functor, for every $(\mathbb{T}, V)$-category $X$.

(3) Let $u \in V$ satisfying $u \cdot !=\xi \cdot T u$. Then $T(r \otimes u)=(T r) \otimes u$, for every $V$-relation $r: X \rightarrow Y$.

Proof. The first assertion is [Hof11, Proposition 1.4(1)]. To see (2), assume that $u \in V$ with $u \cdot ! \geq \xi \cdot T u$. Let $(X, a)$ be a $(\mathbb{T}, V)$-category, $\mathfrak{x} \in T X$ and $x \in X$. Considering the map $X \stackrel{!}{\rightarrow} 1 \stackrel{u}{\rightarrow} V$, we have to show that

$$
a(\mathfrak{x}, x) \leq a(\mathfrak{x}, x) \otimes \operatorname{hom}(T(u \cdot !)(\mathfrak{x}), u),
$$

which follows immediately from $u \cdot ! \geq \xi \cdot T u$. Finally, to prove (3), let $r: X \rightarrow Y$ be a $V$-relation and $u \in V$ with $u \cdot !=\xi \cdot T u$. Note that the $V$-relation $r \otimes u: X \rightarrow Y$ is given by

$$
X \times Y \stackrel{r}{\longrightarrow} V \stackrel{\left\langle 1_{V}, u ! !\right.}{\longrightarrow} V \times V \stackrel{\otimes}{\longrightarrow} V .
$$

Hence, applying the Set-functor $T$ to the functions $r: X \times Y \rightarrow V$ and $r \otimes u: X \times Y \rightarrow V$, we obtain

$$
\begin{aligned}
\xi \cdot T(r \otimes u) & =\xi \cdot T(\otimes) \cdot T\left\langle 1_{V}, u \cdot !\right\rangle \cdot T r \\
& =\otimes \cdot(\xi \times \xi) \cdot \operatorname{can}_{X, Y} \cdot T\left\langle 1_{V}, u \cdot !\right\rangle \cdot T r \\
& =\otimes \cdot\langle\xi, u \cdot ! \cdot \xi\rangle \cdot T r \\
& =\otimes \cdot\left\langle 1_{V}, u ! !\right\rangle \cdot \xi \cdot T r .
\end{aligned}
$$

Therefore, returning to $V$-relations, we conclude that $T(r \otimes u)=(T r) \otimes u$.

Remark 7.3. If $T 1=1$, then $u \cdot !=\xi \cdot T u$ for every $u \in V$.

In order to guarantee Assumptions 5.5 (1), we need an extra condition on $\xi$.

Proposition 7.4. Assume that

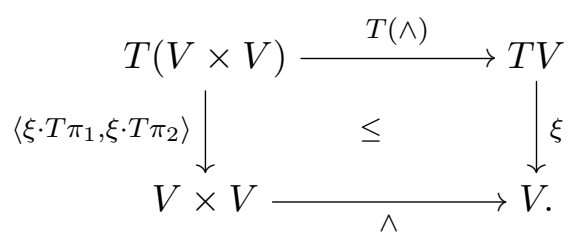


Then, for all $V$-relations $r: X \rightarrow X^{\prime}$ and $s: Y \rightarrow Y^{\prime}$,

$$
\begin{aligned}
& T(X \times Y) \stackrel{\operatorname{can}_{X, Y}}{\longrightarrow} T X \times T Y \\
& T(r \otimes s) \downarrow \quad \geq \quad f^{T r \otimes T s} \\
& T\left(X^{\prime} \times Y^{\prime}\right) \underset{\operatorname{can}_{X^{\prime}, Y^{\prime}}}{\longrightarrow} T X^{\prime} \times T Y^{\prime} .
\end{aligned}
$$

Proof. First we note that, from the preservation of weak pullbacks by $T$, it follows that the commutative diagram

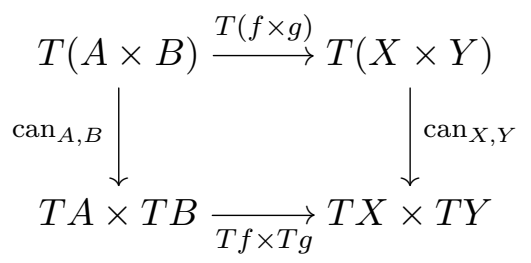

is also a weak pullback.

Let $\mathfrak{w} \in T(X \times Y), \mathfrak{x}^{\prime} \in T X^{\prime}$ and $\mathfrak{y}^{\prime} \in T Y^{\prime}$. Put $(\mathfrak{x}, \mathfrak{y})=\operatorname{can}_{X, Y}(\mathfrak{w})$. By the definition of the extension of $T$ and since $V$ is a Heyting algebra,

$$
\operatorname{Tr}\left(\mathfrak{x}, \mathfrak{x}^{\prime}\right) \wedge T s\left(\mathfrak{y}, \mathfrak{y}^{\prime}\right)=\bigvee\left\{\xi \cdot T r\left(\mathfrak{w}_{1}\right) \wedge \xi \cdot T s\left(\mathfrak{w}_{2}\right) \mid \begin{array}{l}
\mathfrak{w}_{1} \in T\left(X \times X^{\prime}\right): \mathfrak{w}_{1} \mapsto \mathfrak{x}, \mathfrak{w}_{1} \mapsto \mathfrak{x}^{\prime} \\
\mathfrak{w}_{2} \in T\left(Y \times Y^{\prime}\right): \mathfrak{w}_{2} \mapsto \mathfrak{y}, \mathfrak{w}_{2} \mapsto \mathfrak{y}^{\prime}
\end{array}\right\}
$$

Note that in

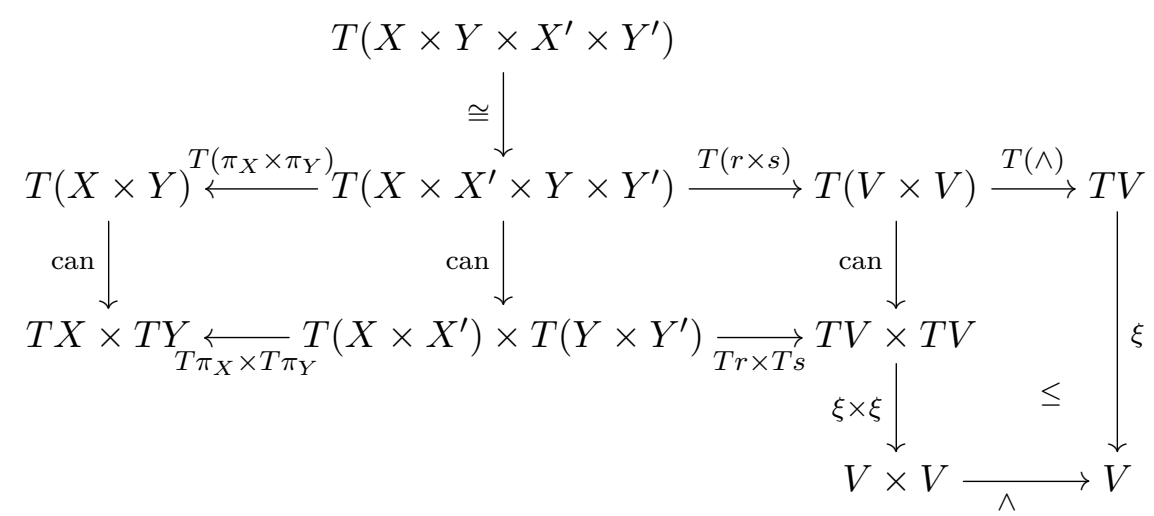

the left hand side is a weak pullback, the middle diagram commutes, and in the right hand side we have "lower path" $\leq$ "upper path" as indicated. Therefore, for such $\mathfrak{w}_{1} \in T\left(X \times X^{\prime}\right)$ and $\mathfrak{w}_{2} \in T\left(Y \times Y^{\prime}\right)$, there exists some $\mathfrak{v} \in T\left(X \times X^{\prime} \times Y \times Y^{\prime}\right)$ which projects to $\mathfrak{w} \in T(X \times Y)$ and to $\left(\mathfrak{w}_{1}, \mathfrak{w}_{2}\right) \in T\left(X \times X^{\prime}\right) \times T\left(Y \times Y^{\prime}\right)$. Hence, taking also into account the definition of the $V$-relation $T(r \otimes s)$,

$$
\begin{aligned}
\operatorname{Tr}\left(\mathfrak{x}, \mathfrak{x}^{\prime}\right) \wedge T s\left(\mathfrak{y}, \mathfrak{y}^{\prime}\right) & \leq \bigvee\left\{\xi \cdot T(\wedge) \cdot T(r \times s)(\mathfrak{v}) \mid \mathfrak{v} \in T\left(X \times Y \times X^{\prime} \times Y^{\prime}\right) ; \begin{array}{l}
\mathfrak{v} \mapsto \mathfrak{w} \\
\mathfrak{v} \mapsto \mathfrak{x}^{\prime}, \mathfrak{v} \mapsto \mathfrak{y}^{\prime}
\end{array}\right\} \\
& \leq \bigvee\left\{T(r \otimes s)\left(\mathfrak{w}, \mathfrak{w}^{\prime}\right) \mid \mathfrak{w}^{\prime} \in T\left(X^{\prime} \times Y^{\prime}\right), \operatorname{can}_{X^{\prime}, Y^{\prime}}\left(\mathfrak{w}^{\prime}\right)=\left(\mathfrak{x}^{\prime}, \mathfrak{y}^{\prime}\right)\right\} .
\end{aligned}
$$

Remark 7.5. We note that the inequality

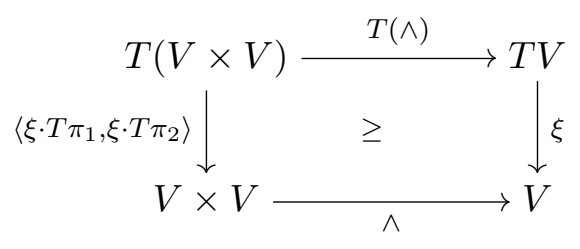


is always true.

Corollary 7.6. If the quantale $V$ satisfies Assumption 5.5 (2) and the diagrams

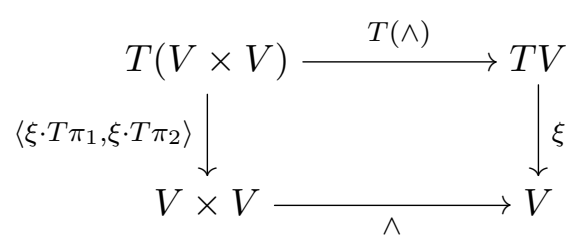

and

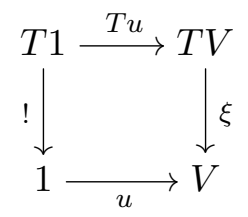

commute, for all $u \in V$, then all Assumptions 5.5 are satisfied.

Let $\mathbb{T}$ be the ultrafilter monad $\mathbb{U}=(U, m, e)$. Then, when $V$ is any of the quantales listed above but $\Delta$, all the needed conditions are satisfied. Therefore, in particular we can conclude that:

Examples 7.7. (1) The category Top $=(\mathbb{U}, 2)$-Cat of topological spaces and continuous maps is weakly cartesian closed (as shown by Rosický in [Ros99]).

(2) The category $\mathbf{A p p}=\left(\mathbb{U}, P_{+}\right)$-Cat of approach spaces and non-expansive maps is weakly cartesian closed.

(3) In fact, for each continuous quantale structure on the lattice $([0,1], \leq) \simeq([0, \infty], \geq)$, $(\mathbb{U},[0,1])$-Cat is weakly cartesian closed. In particular, the category of non-Archimedean approach spaces and non-expansive maps studied in [CVO17] is weakly cartesian closed.

(4) If $V$ is a completely distributive complete lattice with $\otimes=\wedge$, then, with

$$
\xi: U V \rightarrow V, \mathfrak{x} \mapsto \bigwedge_{A \in \mathfrak{x}} \bigvee A,
$$

all the conditions of Theorem 6.3 are satisfied (see [Hof07, Theorem 3.3]) and therefore $(\mathbb{U}, V)$-Cat is weakly cartesian closed. In particular, with $V=P 2$ being the powerset of a 2-element set, we obtain that the category BiTop of bitopological spaces and bicontinuous maps is weakly cartesian closed (see [HST14]).

Remark 7.8. For $V=\Delta$ the quantale of distribution functions, we do not know whether there is an appropriate compact Hausdorff topology $\xi: U V \rightarrow V$ satisfying the conditions of this section.

Now let $\mathbb{T}$ be the free monoid monad $\mathbb{W}=(W, m, e)$. For each quantale $V$, we consider

$$
\xi: W V \rightarrow V,\left(v_{1}, \ldots, v_{n}\right) \mapsto v_{1} \otimes \cdots \otimes v_{n},() \mapsto k
$$

which induces the extension $W: V$-Rel $\rightarrow V$-Rel sending $r: X \rightarrow Y$ to the $V$-relation $W r$ : $W X \rightarrow W Y$ given by

$$
W r\left(\left(x_{1}, \ldots, x_{n}\right),\left(y_{1}, \ldots, y_{m}\right)\right)= \begin{cases}r\left(x_{1}, y_{1}\right) \otimes \cdots \otimes r\left(x_{n}, y_{n}\right) & \text { if } n=m \\ \perp & \text { if } n \neq m .\end{cases}
$$

The category (W, 2)-Cat is equivalent to the category MultiOrd of multi-ordered sets and their morphisms (see [HST14]), more generally, (WW, $V$ )-categories can be interpreted as multi- $V$-categories and their morphisms. The representable multi-ordered sets are precisely the ordered monoids, which is a special case of [Her00, Her01] describing monoidal categories as representable multi-categories (see also [CCH15]). We recall that the separated injective multi-ordered sets are precisely the quantales (see [LBKR12] and also [Sea10]), and we conclude:

Proposition 7.9. Every quantale is exponentiable in MultiOrd.

Theorem 7.10. If the quantale $V$ is a frame, then (WW, $V$ )-Cat is weakly cartesian closed. In particular, MultiOrd is weakly cartesian closed. 
Finally, for a monoid $(H, \cdot, h)$, we consider the monad $\mathbb{H}=(-\times H, m, e)$, with $m_{X}: X \times H \times H \rightarrow$ $X \times H$ given by $m_{X}(x, a, b)=(x, a \cdot b)$ and $e_{X}: X \rightarrow X \times H$ given by $e_{X}(x)=(x, h)$. Here we consider

$$
\xi: V \times H \rightarrow V,(v, a) \mapsto v,
$$

which leads to the extension $-\times H: V$-Rel $\rightarrow V$-Rel sending the $V$-relation $r: X \rightarrow Y$ to the $V$-relation $r \times H: X \times H \rightarrow Y \times H$ with

$$
r \times H((x, a),(y, b))= \begin{cases}r(x, y) & \text { if } a=b, \\ \perp & \text { if } a \neq b .\end{cases}
$$

In particular, (HI, 2)-categories can be interpreted as $H$-labelled ordered sets and equivariant maps.

For every quantale $V$ and every $v: 1 \rightarrow V$, the diagrams
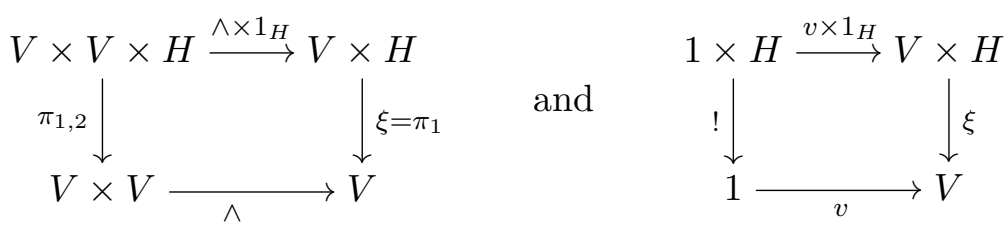

commute, therefore we obtain:

Theorem 7.11. For every quantale $V$ satisfying Assumption 5.5 (2), the category (HI, $V$ )-Cat is weakly cartesian closed.

\section{REFERENCES}

[AHS90] Jiří Adámek, Horst Herrlich, and George E. Strecker. Abstract and concrete categories: The joy of cats. Pure and Applied Mathematics (New York). John Wiley \& Sons Inc., New York, 1990. Republished in: Reprints in Theory and Applications of Categories, No. 17 (2006) pp. 1-507.

[AR18] Jiří Adámek and Jiří Rosický. How nice are free completions of categories? Technical report, 2018, arXiv: 1806.02524 [math.CT].

[BBS04] Andrej Bauer, Lars Birkedal, and Dana S. Scott. Equilogical spaces. Theoretical Computer Science, 315(1):35-59, 2004.

[CCH15] Dimitri Chikhladze, Maria Manuel Clementino, and Dirk Hofmann. Representable (T, $\mathcal{V})$-categories. Applied Categorical Structures, 23(6):829-858, January 2015, eprint: http://www.mat.uc.pt/preprints/ $\mathrm{ps} / \mathrm{p} 1247 . \mathrm{pdf}$

[CH03] Maria Manuel Clementino and Dirk Hofmann. Topological features of lax algebras. Applied Categorical Structures, 11(3):267-286, June 2003, eprint: http://www.mat.uc.pt/preprints/ps/p0109.ps

[CH06] Maria Manuel Clementino and Dirk Hofmann. Exponentiation in $V$-categories. Topology and its Applications, 153(16):3113-3128, October 2006.

[CH09] Maria Manuel Clementino and Dirk Hofmann. Lawvere completeness in topology. Applied Categorical Structures, 17(2):175-210, August 2009, arXiv:0704.3976 [math.CT].

[CH17] Maria Manuel Clementino and Dirk Hofmann. The Rise and Fall of $V$-functors. Fuzzy Sets and Systems, 321:29-49, August 2017, eprint: http://www.mat.uc.pt/preprints/ps/p1606.pdf

[CHJ14] Maria Manuel Clementino, Dirk Hofmann, and George Janelidze. The monads of classical algebra are seldom weakly cartesian. Journal of Homotopy and Related Structures, 9(1):175-197, November 2014, eprint: http://www.mat.uc.pt/preprints/ps/p1246.pdf.

[CHT03] Maria Manuel Clementino, Dirk Hofmann, and Walter Tholen. Exponentiability in categories of lax algebras. Theory and Applications of Categories, 11(15):337-352, 2003, eprint: http://www.mat.uc.pt/ preprints/ps/p0302.pdf.

[CT03] Maria Manuel Clementino and Walter Tholen. Metric, topology and multicategory — a common approach. Journal of Pure and Applied Algebra, 179(1-2):13-47, April 2003.

[CVO17] Eva Colebunders and Karen Van Opdenbosch. Topological properties of non-Archimedean approach spaces. Theory and Applications of Categories, 32(41):1454-1484, 2017.

[Fau55] William M. Faucett. Compact semigroups irreducibly connected between two idempotents. Proceedings of the American Mathematical Society, 6(5):741-747, May 1955. 
[Her00] Claudio Hermida. Representable multicategories. Advances in Mathematics, 151(2):164-225, May 2000.

[Her01] Claudio Hermida. From coherent structures to universal properties. Journal of Pure and Applied Algebra, 165(1):7-61, November 2001.

[Hof06] Dirk Hofmann. Exponentiation for unitary structures. Topology and its Applications, 153(16):3180-3202, October 2006.

[Hof07] Dirk Hofmann. Topological theories and closed objects. Advances in Mathematics, 215(2):789-824, November 2007.

[Hof11] Dirk Hofmann. Injective spaces via adjunction. Journal of Pure and Applied Algebra, 215(3):283-302, March 2011, arXiv:0804.0326 [math.CT]

[Hof14] Dirk Hofmann. The enriched Vietoris monad on representable spaces. Journal of Pure and Applied Algebra, 218(12):2274-2318, December 2014, arXiv:1212.5539 [math.CT]

[HR13] Dirk Hofmann and Carla D. Reis. Probabilistic metric spaces as enriched categories. Fuzzy Sets and Systems, 210:1-21, January 2013, arXiv:1201.1161 [math.GN].

[HS15] Dirk Hofmann and Gavin J. Seal. Exponentiable approach spaces. Houston Journal of Mathematics, 41(3):1051-1062, 2015, arXiv:1304.6862 [math.GN]

[HST14] Dirk Hofmann, Gavin J. Seal, and Walter Tholen, editors. Monoidal Topology. A Categorical Approach to Order, Metric, and Topology, volume 153 of Encyclopedia of Mathematics and its Applications. Cambridge University Press, Cambridge, July 2014. Authors: Maria Manuel Clementino, Eva Colebunders, Dirk Hofmann, Robert Lowen, Rory Lucyshyn-Wright, Gavin J. Seal and Walter Tholen.

[HT10] Dirk Hofmann and Walter Tholen. Lawvere completion and separation via closure. Applied Categorical Structures, 18(3):259-287, November 2010, arXiv:0801.0199 [math.CT]

[Law73] F. William Lawvere. Metric spaces, generalized logic, and closed categories. Rendiconti del Seminario Matemàtico e Fisico di Milano, 43(1):135-166, December 1973. Republished in: Reprints in Theory and Applications of Categories, No. 1 (2002), 1-37.

[LBKR12] Joachim Lambek, Michael Barr, John F. Kennison, and Robert Raphael. Injective hulls of partially ordered monoids. Theory and Applications of Categories, 26(13):338-348, 2012.

[Low97] Robert Lowen. Approach Spaces: The Missing Link in the Topology-Uniformity-Metric Triad. Oxford Mathematical Monographs. Oxford University Press, Oxford, 1997.

[MS57] Paul S. Mostert and Allen L. Shields. On the structure of semi-groups on a compact manifold with boundary. Annals of Mathematics. Second Series, 65(1):117-143, January 1957.

[MST06] Mojgan Mahmoudi, Christoph Schubert, and Walter Tholen. Universality of coproducts in categories of lax algebras. Applied Categorical Structures, 14(3):243-249, June 2006.

[Rib18] Willian Ribeiro. On generalized equilogical spaces. Technical Report 18-50, Department of Mathematics, University of Coimbra, 2018, arXiv:1811.08240 [math.CT].

[Ros99] Jiří Rosický. Cartesian closed exact completions. Journal of Pure and Applied Algebra, 142(3):261-270, October 1999.

[Sea10] Gavin J. Seal. Order-adjoint monads and injective objects. Journal of Pure and Applied Algebra, 214(6):778-796, June 2010

[Tho09] Walter Tholen. Ordered topological structures. Topology and its Applications, 156(12):2148-2157, July 2009.

CMuC, Department of Mathematics, University of Coimbra, 3001-501 Coimbra, Portugal

E-mail address: mmc@mat.uc.pt

Cidma, Department of Mathematics, University of Aveiro, 3810-193 Aveiro, Portugal

E-mail address: dirk@ua.pt

CMuC, Department of Mathematics, University of Coimbra, 3001-501 Coimbra, Portugal

E-mail address: willian.ribeiro.vs@gmail.com 